\title{
Phylogenetic characterization of norovirus strains detected from sporadic gastroenteritis in Seoul during 2014-2016
}

Young Eun Kim¹,2, Miok Song ${ }^{1}$, Jaein Lee ${ }^{1}$, Hyun Jung Seung ${ }^{1}$, Eun-Young Kwon ${ }^{1}$, Jinkyung Yu', Youngok Hwang ${ }^{1}$, Taeho Yoon', Tae Jun Park ${ }^{2}$ and In Kyoung Lim² ${ }^{2 *}$

\begin{abstract}
Background: Phylogenetic analysis of norovirus (NoV) is efficient for tracking NoV transmission. To determine the widespread NoV strains in Seoul, we conducted an extensive phylogenetic characterization of NoV-positives from 1659 diarrheal specimens collected in 2014-2016 for the Seoul NoV-surveillance.

Results: When the large numbers of NoV partial VP1 genome sequences were analyzed in acute gastroenteritis patients along with the phylogenetic characterization, we could identify molecular epidemiologic patterns based on the genetic characteristics of sporadic NoV strains circulating in Seoul, which could provide a detailed description of the genome-wide and community-wide NoV evolution in each genotype. The average NoV detection rate in our study period was $16.34 \%$ that was increased by $7.44 \%$ from $13.17 \%$ in 2014 to $20.61 \%$ in 2016 . Prevalence of NoV Gl and Gll was 4.43\% and 93.36\%, respectively, and the Gll.4, Gll.17, and Gll.3 were found to be the major type among 17 genotypes of NoV. The most prevalent one was Gll.4 (50.92\%) that was followed by GIl.17 (18.08\%) and Gll.3 (9.96\%). According to an extensive phylogenetic analysis based on partial VP1 sequences of 1008 NoV (276 sporadic, 518 outbreak and 214 reference), pandemic strains of Gll.17, Gll.4 and Gll.3 have emerged in succession during the 20142016 Seoul NoV-surveillance. Gll.17 emerged as Gll.17|Kawasaki323 in 2014, and became the predominant genotype in 2015 with Gll.17|2014_Kawasaki lineages (CUHK-NS-616/Kawasaki308). The formerly predominant Gll.4 remained high-level with GII.4|2012_Sydney in 2014 and internally replaced to GII.4|2016_Kawasaki194 lineage (NOR-2565/ NOR-2558/OH16002) that caused the sporadic NoV explosion since December 2015. Sporadically prevalent GIl.3|Hu/ Aichio334-13/2013 failed to develop any outbreaks, whereas sporadic GII.3|Hu/3-28/2015/HNZZ/CHN caused heavy outbreaks in Seoul without preparation time since November 2016.
\end{abstract}

Conclusions: This is the first extensive phylogenetic study revealing the important events of NoV strains circulating in Seoul. Particularly, our study period from 2014 to 2016 was very dynamic with the emergences of the three main NoV strains (GIl.17|2014_Kawasaki, GII.4|2016_Kawasaki194 and GII.3|Hu/3-28/2015/HNZZ/CHN) every year. We are sure that it is hard to detect above findings by simple conventional analysis. Our present study reports a future paradigm of the NoV molecular epidemiology, which might be highly valuable to track new strains and predict oncoming outbreaks.

Keywords: Norovirus-GII.4, -GII.17,-GIl.3 surveillance, Virus epidemiology, Phylogenetic analysis, Molecular characterization, Sporadic AGE (acute gastroenteritis)

\footnotetext{
*Correspondence: iklim@ajou.ac.kr

2 Department of Biochemistry and Molecular Biology, Ajou University School of Medicine and Graduate School of Medicine, 164 Worldcup-ro, Yeongtong-gu, Suwon 16499, Republic of Korea

Full list of author information is available at the end of the article
} 


\section{Background}

Acute gastroenteritis (AGE) causes one of the major public health problems [1], and NoV has been reported as the most common cause of AGE [2]. NoV is a nonenveloped, positive-sense, single-stranded RNA virus with a linear genome (7.5-7.7 kb), which belongs to the family Caliciviridae with three open reading frames (ORFs) encoding nine structural and nonstructural proteins [3-5]. ORF1 encodes nonstructural proteins such as NTPase, protease, and RNA-dependent RNA polymerase (RdRp). ORF2 overlaps ORF1 by a short region and encodes the major capsid protein, VP1. ORF3 encodes the minor capsid protein, VP2 [6]. NoVs are highly diverse and currently sub-divided into six genomic groups (GI/GII/GIII/GIV/GV/GVI) with more than 40 genotypes based on their VP1 sequences $[7,8]$.

It has been reported that NoV caused at least six pandemics of AGE (defined as taking place on at least three continents over a similar time-frame) since 1995; 1995-1996 (GII.4|US95_96), 2002-2003 (GII.4|Farmington Hills), 2004-2005 (GII.4|Hunter), 2006-2007 (GII.4|2006a_Yerseke and GII.4|2006b_Den Haag), 2009-2010 (GII.4|New Orleans) and 2012-2013 (GII.4|Sydney) [8]. NoV exhibits over 40 genotypes cocirculating within the population, however, GII.4 has emerged only as novel variants about every $2-4$ years, massive outbreaks, and pandemics [9]. Like influenza virus, population immunity may drive the evolution of $\mathrm{NoV}$ and the emergences of its new variants [10], which undergoes genetic and antigenic evolution through accumulation of point mutations and intra- and intergenotype recombinations [11].

As awareness and knowledge about the growth of Seoul NoV epidemiology, the question has been raised how to effectively track the emergence of new NoV strains, and how to monitor the spread of them. We, therefore, tried to create a more extensive phylogenetic characterization of our data obtained from the Seoul NoV surveillance. This NoV-surveillance system aimed at controlling the spread of future NoV outbreaks by monitoring the circulating strains. Here, we presented the widespread- and newly emerged-NoV strains in Seoul and tried to characterize their molecular epidemiology in the 2014-2016 Seoul surveillance. During the 3-year study period, we have observed novel epidemic strains found in global distribution, however, their sub-lineages showed different scale and impact in distribution or prevalence along with co-circulating strains in Seoul. Here, we also reported sporadic strains developed into outbreaks in our NoV-surveillance system. Total 1008 sequences were analyzed phylogenetically in the five different NoV models (GI/GII.4/GII.17/ GII.3/other types of GII).

\section{Methods}

Ethics statement

All the processes from sample collection to diagnosis of NoV were followed by National Norovirus Surveillance System in Korea (K-CaliciNet) and the "Guideline for water and foodborne diseases prevention and control" [12] under the Korean "Enforcement Regulations of the Infectious Disease Control and Prevention Act". All data were handled based on the Korea Centers for Disease Control and Prevention (KCDC) regulations. Present study was carried out after the diagnosis of NoV as an anonymous epidemiological data and a phylogenetic characterization of NoV sequences. According to the Human Subjects Institutional Review Board (IRB) of Korea National Institute for Bioethics Policy, our present study is excluded from the subject to deliberation.

\section{Specimens and diagnosis}

Diarrheal fecal specimens, total 1659 , were collected from AGE patients with age range 0-84 from ten local hospitals in Seoul during 2014-2016 (Fig. 1). The samples were weekly collected and pretreated on arrival to test by qRTPCR using PowerCheck ${ }^{\mathrm{TM}}$ Norovirus GI/GII (Kogene, Korea). For phylogenetic analysis, partial regions of VP1 for GI and GII genotypes were amplified by RT-PCR and semi-nested RT-PCR with the primers described in Table 1 [13] using the HyQ ${ }^{\mathrm{TM}}$ One-step RT-PCR kit (SNC, Korea). To apply one-step RT-PCR, the specific primer pairs (GI-F1M/GI-R1M and GII-F1M/GII-R1M) targeting VP1 in ORF2 were applied. Semi-nested PCR was performed using the one-step RT-PCR product $(2 \mu \mathrm{l})$ and the primers (GI-F2/GI-R1M and GII-F3/GII-R1M). Before we request DNA sequencing in Macrogen, Inc. (Seoul, Korea), the RT-PCR products were purified using the QIAquick Gel Extraction Kit (Qiagen, Hilden, Germany). Analysis of nucleotide sequences was carried out by Macrogen using the Big Dye Dideoxy cycle sequencing kit and the ABI PRISM 3730XL Analyzer (Applied Biosystem, USA). The diagnostic practice for NoV detection was conducted according to the guidelines of the KCDC [12] and the manufacturer's instructions.

In our present study, total 4073 diarrheal specimens (1659 sporadic, 2414 outbreak) of AGE patients could cover in part the population in Seoul to represent $\mathrm{NoV}$ trend in Korea. In addition, we have expanded the study period of Seoul NoV epidemicity from the 3 year (20142016) to 10 year (2007-2016) by reanalyzing the unreported-NoV surveillance data (Fig. 3).

To detect sporadic strains developed into outbreaks in the Seoul NoV-surveillance system, 518 strains out of 2414 AGE patients obtained from outbreaks in Seoul during January 2014-June 2017 were compared with 276 strains out of 1659 AGE patients obtained from 


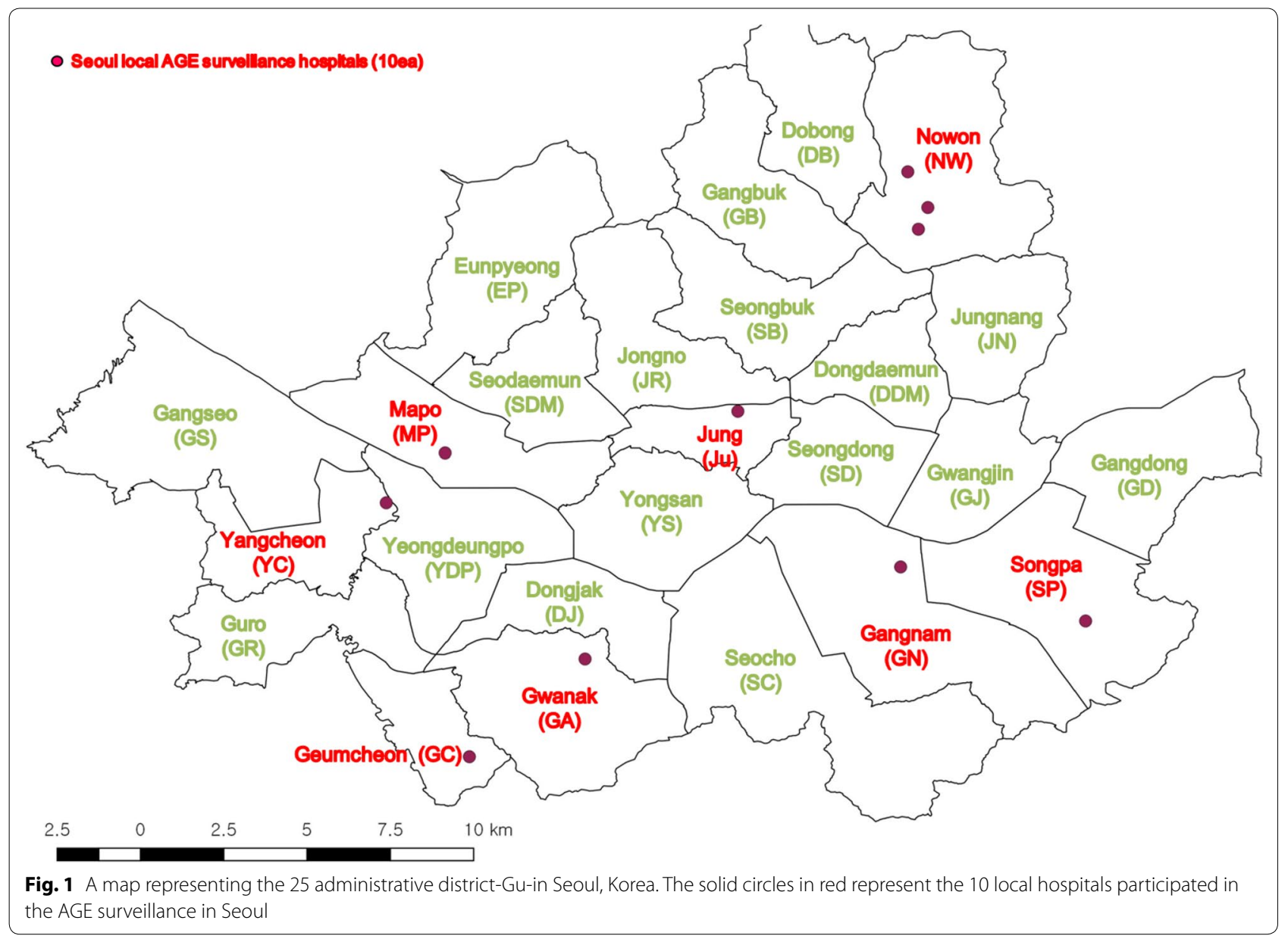

Table 1 Primer sequences for norovirus detection

\begin{tabular}{llllll}
\hline Virus & Primer & Sequence $\left(\mathbf{5}^{\prime} \boldsymbol{\rightarrow} \mathbf{3}^{\prime}\right)$ & Position $(\mathbf{n t})$ & Size (bp) & References \\
\hline Norovirus Gl & GI-F1M & CTGCCCGATTYGTAAATGATGAT & $5336-5359$ & 314 & {$[13]$} \\
& GI-R1M & CCAACCCARCCATTRTACATYTG & $5643-5665$ & & \\
Norovirus GII & GI-F2 & ATGATGATGGCGTCTAAGGACGC & $5352-5374$ & 513 & {$[13]$} \\
& GII-F1M & GGGAGGGCGATCGCAATCT & $5049-5067$ & $5367-5389$ & \\
& GII-R1M & CCRCCIGCATRICCRTTRTACAT & $5077-5100$ & \\
\hline
\end{tabular}

a Carried out by RT-PCR and semi-nested PCR

the surveillance in January 2014-December 2016. To examine the phylogenetic relationship with reference strains, 126 candidate standard strains (Additional file 1: Table S1) and 88 global strains (selected only as highly similar to our strains among updated NCBI sequences in each genotype) of NoV GI and GII were collected from NoroNet and GenBank, and then phylogenetically analyzed above-mentioned $794(518+276)$ strains in the five NoV models, GI/GII.4/GII.17/GII.3/other types of GII (Fig. 2). To investigate presence or absence of unreported-NoV epidemic curves, we reanalyzed the data $[14,15]$ that had been previously obtained from the Seoul NoV-surveillance system in 2007-2013 (Fig. 3).

\section{Phylogenetic analysis}

With rapid accumulation of huge norovirus sequence data [16] and an attempt to establish a unified norovirus classification in the CDC [17], the complete VP1 was replaced by the partial nucleotide sequence of a highlyvariable N-terminal region in the VP1 (277-nucleotides). 


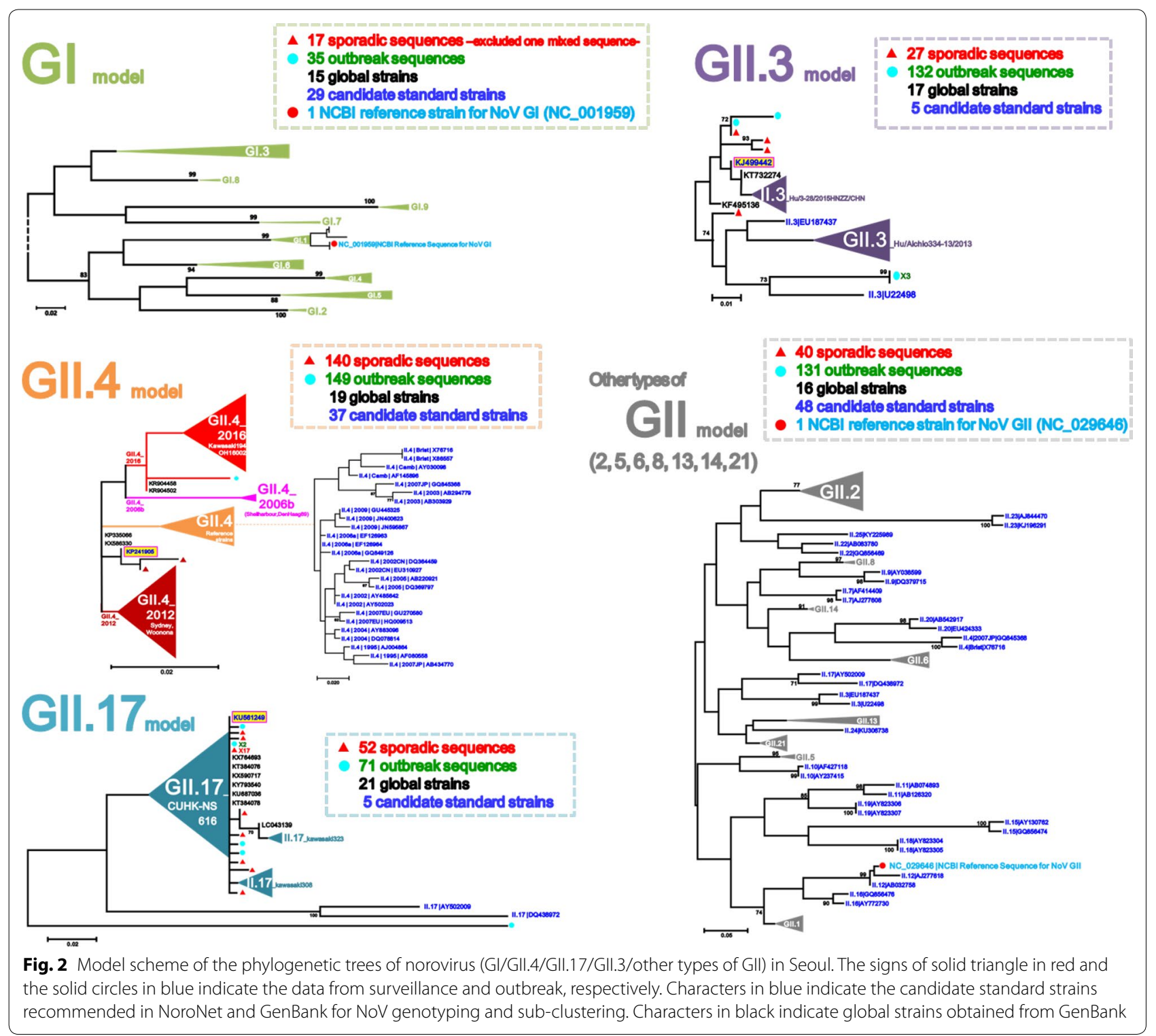

Therefore, the partial VP1 capsid region in ORF2 has routinely been used since 2006 to investigate genotyping and presence of norovirus variants by using a web-based automated typing tool [18]. For convenience of analysis, the primer sets [13] targeting a partial VP1 region have been recommended for PCR cloning and sequencing in the National Norovirus Surveillance Guideline in Korea [12]. In the five phylogenetic trees (GI/GII.4/ GII.17/GII.3/other types of GII), 276 sporadic sequences and 518 outbreaks were aligned with each candidate standard strains (Additional file 1: Table S1) and additional global strains (Fig. 2). Phylogenetic analysis was performed using MEGA7.0 (Fig. 2) based on the partial VP1 sequences (289 nucleotides at the 5360-5648 with reference to NC_001959 for GI, and 279-293 nucleotides from the 5085 to 5363-5377 with reference to NC_029646 for GII). All aligned sequences were trimmed at the above-mentioned nucleotide positions as that of each NoV GI and GII strain. Maximum Likelihood phylogenetic trees constructed in MEGA 7.0 program were inferred from the 1000 replicates based on the TamuraNei model with a bootstrap consensus tree, and the bootstrap values were given above $70 \%$.

\section{Results}

Prevalence of NoV

NoV was detected in the 271 specimens out of 1659 (16.34\%) and the annual detection rate was increased 


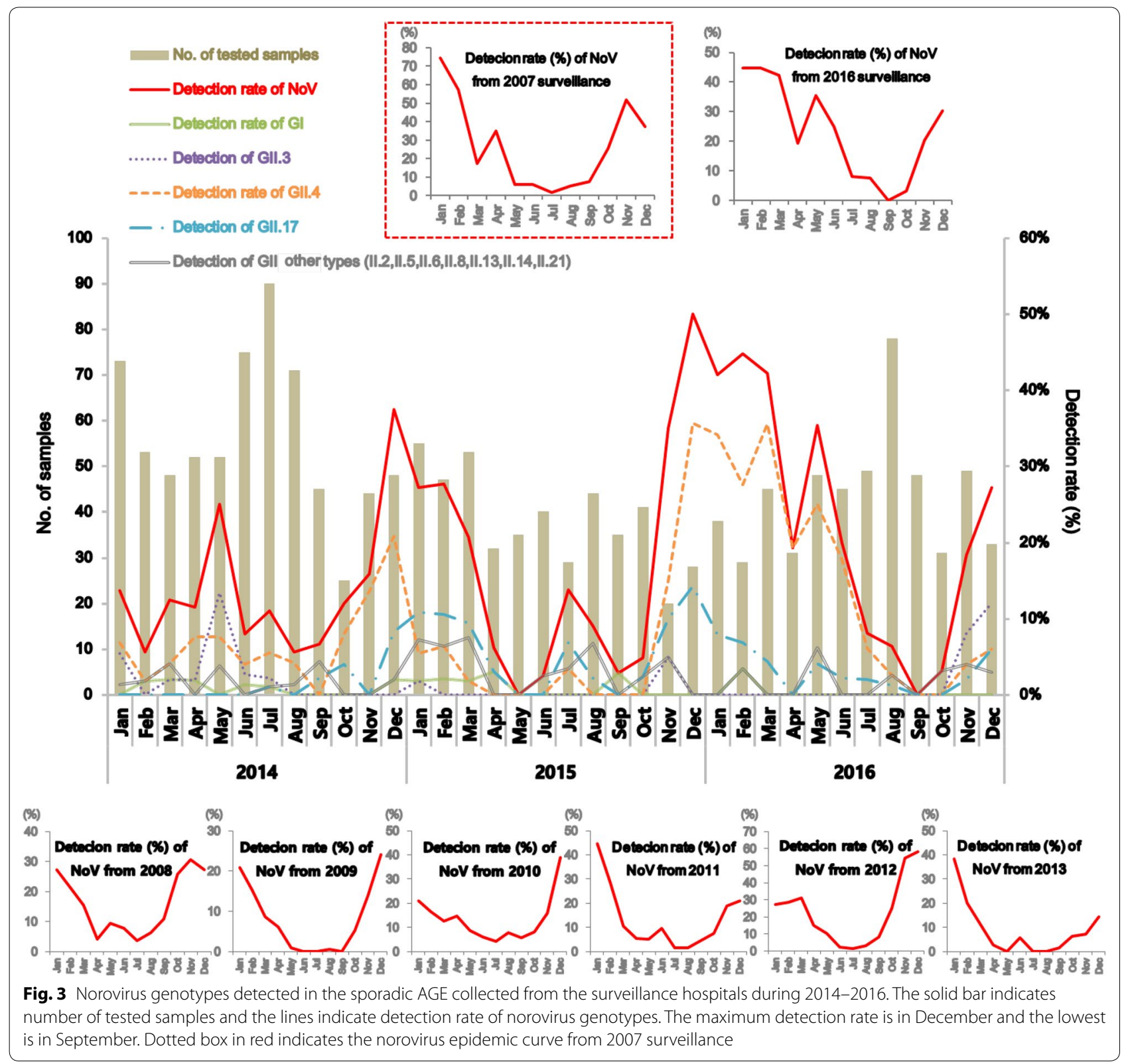

by $7.44 \%$ from $13.17 \%$ in 2014 to $20.61 \%$ in 2016 ; presence in 89 samples among 676 in 2014, 74 among 459 samples in 2015, and 108 among 524 samples in 2016. In monthly surveillance over the 3 years (2014-2016), NoV was detected almost all year around except in May, 2015 and September, 2016 (Fig. 3). Genotyping was performed with $271 \mathrm{NoV}$-positive fecal specimens and found that prevalence of GI (12/271 positives) was $4.43 \%$, that of GII (253/271 positives) was $93.36 \%$, and GI and GII combined was $2.21 \%$ (6/271 positives) (Table 2$)$. The most prevalent strain of NoV was found to be GII.4; prevalence of GII.4 was 50.92\% (138/271 positives), GII.17 was $18.08 \%$ (49/271 positives), GII.3 was $9.96 \%$ (27/271 positives), GII.6 was 5.54\% (15/271 positives), GII.21 was $4.06 \%$ (11/271 positives), GII.13 was $2.58 \%$ (7/271 positives), GII.2 was $1.48 \%$ (4/271 positives), and the rarely detected GII.5, GII.8, and GII.14 was positive only one fecal specimen out of 271 (0.37\%) (Fig. 3 and Table 2).

\section{Monthly distribution of NoV epidemicity}

Monthly prevalence trend of the NoV genotypes was variable (Fig. 3 and Table 3). To compare the previous data obtained from the Seoul NoV-surveillance during the past 7 years with our present study, the highest and 
Table 2 Genotypes of sporadic noroviruses and number of samples identified from the Seoul surveillance test during 2014-2016

\begin{tabular}{|c|c|c|c|c|c|}
\hline Enteric virus & Genotype & No. of samples & Genotype & No. of samples & Total \\
\hline \multirow[t]{4}{*}{ Norovirus Gl (no. of single Gl genotype) } & $\mathrm{Gl} .1$ & 0 & $\mathrm{Gl} .5$ & 2 & \multirow[t]{4}{*}{12} \\
\hline & $\mathrm{Gl} .2$ & 1 & $\mathrm{Gl} .6$ & 1 & \\
\hline & $\mathrm{Gl} .3$ & 3 & $\mathrm{Gl} .7$ & 1 & \\
\hline & $\mathrm{Gl} .4$ & 3 & Mixed & 1 & \\
\hline \multirow[t]{5}{*}{ Norovirus GII (no. of single Gll genotype) } & Gll.2 & 4 & Gll.8 & 1 & \multirow[t]{5}{*}{253} \\
\hline & Gll.3 & 27 & Gll.13 & 7 & \\
\hline & Gll.4 & 138 & Gll.14 & 1 & \\
\hline & Gll.5 & 1 & Gll.17 & 49 & \\
\hline & Gll.6 & 15 & GIl.21 & 10 & \\
\hline \multirow[t]{2}{*}{ Norovirus Gll.4 (no. of single Gll4 genotype) } & Gll.4|2006b & 1 & Gll.4|2016 & 70 & \multirow[t]{2}{*}{138} \\
\hline & Gll.4|2012 & 67 & (GI \& Gll.4|2012) & (2) & \\
\hline \multirow{3}{*}{$\begin{array}{l}\text { Norovirus GI \& GII (no. of Gl and GIl combination } \\
\text { genotype) }\end{array}$} & Gl.1 \& GII.17 & 1 & Gl.5 \& GIl.17 & 1 & \multirow[t]{3}{*}{6} \\
\hline & Gl.3 \& GIl.17 & 1 & Gl.5 \& GII.21 & 1 & \\
\hline & Gl.4 \& Gll.4 & 1 & Gl.6 \& Gll.4 & 1 & \\
\hline
\end{tabular}

Table 3 Distribution of norovirus genotypes detected by surveillance test during 2014-2016

\begin{tabular}{|c|c|c|c|c|c|c|c|c|c|c|c|c|c|c|}
\hline \multirow[t]{2}{*}{ Month } & \multicolumn{14}{|c|}{ No. of strains of norovirus GI and GII from Seoul in 2014-2016 } \\
\hline & GI & I\&II & II.2 & II.3 & II.4 & II.5 & II.6 & II.8 & II.13 & II.14 & II.17 & II.21 & No. of NoVs & No. of tested \\
\hline January & 1 & - & - & 5 & 21 & - & 3 & - & 2 & - & 9 & - & 41 & 166 \\
\hline February & 3 & 2 & - & - & 12 & - & - & 1 & 3 & - & 7 & 1 & 29 & 129 \\
\hline March & 2 & 1 & 1 & 1 & 19 & - & - & - & 2 & - & 7 & 3 & 36 & 146 \\
\hline April & 2 & - & - & 1 & 1 & - & - & - & - & - & 1 & - & 14 & 115 \\
\hline May & - & - & - & 7 & 16 & 1 & 3 & - & - & - & 2 & 1 & 30 & 135 \\
\hline June & 1 & - & - & 2 & 11 & - & - & - & - & - & 1 & 1 & 16 & 160 \\
\hline July & 1 & - & - & 2 & 9 & - & 1 & - & - & - & 4 & 1 & 18 & 168 \\
\hline August & - & - & 1 & - & 5 & - & 1 & - & - & 1 & 2 & 3 & 13 & 193 \\
\hline September & 1 & - & - & - & - & - & 2 & - & - & - & 1 & - & 4 & 128 \\
\hline October & - & - & - & - & 2 & - & 2 & - & - & - & 2 & - & 6 & 97 \\
\hline November & - & 1 & 1 & 5 & 11 & - & 2 & - & - & - & 3 & - & 23 & 113 \\
\hline December & 1 & 2 & 1 & 4 & 22 & - & 1 & - & - & - & 1 & - & 41 & 109 \\
\hline Total & 12 & 6 & 4 & 27 & 138 & 1 & 15 & 1 & 7 & 1 & 49 & 10 & 271 & 1659 \\
\hline
\end{tabular}

the lowest $\mathrm{NoV}$ frequency were recorded, respectively, as $35.60 \%(366 / 1028$ samples) in January and $2.44 \%$ (20/818 samples) in July in the 2007-2011 surveillance [14], and $37.78 \%$ in December (68/180 samples) and $0.86 \%$ in July (1/116 samples) in the 2012-2013 surveillance [15]. In the 2014-2016 surveillance, the highest frequency was found in December (37.61\%; 41/109 samples) and the lowest was in September (3.13\%; $4 / 128$ samples). The overall epidemicity of the Seoul NoV-surveillance recorded in the 2008-2013 and the 2014-2015 had been found as the "W-shape" and the typical "U-shape" curve, respectively, with seasonal variability, however, the surveillance data detected in the 2007 (dotted box in Fig. 3) and the 2016 revealed a novel "right-sided W-shape" rather than the typical "W (or U)-shape" curve. In the novel "right-sided W-shape" curve in 2016, the major genotype was found to be GII.4 that was the same as the main genotype occurred in the 2014 Seoul NoV-surveillance. Compared to the previous data, the abnormally high activity of the NoV found in winter of 2015-2016 and the high peak found in spring of 2016 could be caused the novel "rightsided W-shape" curve recorded in the 2016 Seoul NoVsurveillance. The main genotype detected in the 2015 
surveillance was identified as GII.17 that may cause the typical "W-shape" curve (Fig. 3 and Table 3 ).

\section{Norovirus phylogenetic analysis: GI, GII.4, GII.17, GII.3 and other types of GII (II.2/5/6/8/13/14/21)}

In the GI phylogenetic tree, the 17 sporadic sequences presented a great diversity of seven different clusters (I.1/I.2/I.3/I.4/I.5/I.6/I.7). The dominant genotypes in the sporadic GI were GI.3, GI.5, and GI.4. Six combinations of GI and GII (I.1_II.17/I.3_II.17/I.4_ II.4/I.5_II.17/I.5_II.21/I.6_II.4) were detected in the GI surveillance. In the GI.3, GI.5 and GI.6 clusters, two or four sporadic sequences were sub-divided into two sub-clusters; GI.3|Beijing55042/54108, GI.5|Musgrove/ Babbacombe and GI.6|VA497/Sindlesham. In GI.1 and GI.4 clusters, two and three sporadic sequences were sub-divided into only one sub-cluster (GI.1|KY-89/89/J and GI.4|Valetta). I.2 and I.7 were sub-divided into one sub-cluster (I.2|Southampton and I.7|Winchester) with only one sporadic sequence. The I.8 and I.9 clusters included one and three outbreaks, but not sporadic one (Figs. 3 and 4).

In the most prevalent GII.4 phylogenetic tree, the 140 sporadic sequences were sub-divided into three sub-clusters (GII.4|2006, GII.4|2012 and GII.4|2016). Out of the 140 sporadic sequences, only one sporadic sequence was found in the GII.4|2006 sub-cluster. In the remaining 139 sporadic sequences, 69 sequences were clustered into the GII.4|2012 sub-cluster, and the last 70 sequences were clustered into GII.4|2016 sub-cluster. The three GII.4 sub-clusters showed a significant difference in their collection time and the development of outbreak; GII.4|2016 sporadic NoV was consistently observed after November 2015 and successfully developed into outbreak at the same time. We assumed that there was no preparation time between the sporadic occurrence and the outbreak explosion caused by the GII.4|2016_Kawasaki194 sublineages (ACT6754/NOR-2565/NOR-2558). However, the 69 sporadic NoV in GII.4|2012 (GII.4|2012_Sydney/ Kawasaki194) frequently detected before November 2015 and failed to develop outbreak during the whole study period. Our phylogenetically analysis may demonstrate GII.4|2016_Kawasaki194 emergence very rapidly in Seoul since December 2015, which might form the novel "rightsided W-shape" curve (Figs. 3 and 5).

In the GII.17 phylogenetic tree, the 52 sporadic sequences GII.17|CUHK-NS-616 (KU561249) were clustered into two sub-clusters (GII.17|Kawasaki308/Kawasaki323). The first sporadic GII.17 from our surveillance emerged as GII.17|Kawasaki323 in July 2014. Since we started surveillance in 2015, GII.17 was found to be the predominant genotype with GII.17|2014_Kawasaki lineage (CUHK-NS-616/Kawasaki308) for the first time, which replaced the previously dominant GII.4 genotype. Although occurrence of GII.17 continuously increased from December 2014 to March 2015, it could not overcome the outbreak explosion by GII.4|2016_Kawasaki194 in November 2015 (Figs. 3 and 6).

In the GII.3 phylogenetic tree, the 26 sporadic sequences were clustered into three sub-clusters (GII.3|Hu/Aichio334-13/2013, GII.3|Hu/3-28/2015/ HNZZ/CHN and GII.3/Hu/HKG/2013/CUHKNS-201). In the phylogenetic tree, none of the sporadic GII.3 sequences presented any identical outbreak one, except in an overseas inflow. Although GII.3|Hu/ Aichio334-13/2013 (LC089676) was the most prevalent GII.3 strain in the Seoul NoV-surveillance during 2014-2016, any of the 15 sporadic sequences failed to develop any outbreak. In GII.3|Hu/3-28/2015/HNZZ/ CHN (KY767664) causing the GII.3 explosion in Seoul since November 2016, the first sequences from our surveillance and the outbreak emerged simultaneously in November 2016. We thought that there was no preparation time between the sporadic occurrence and the outbreak associated with the GII.3|Hu/3-28/2015/HNZZ/ CHN strains (Figs. 3 and 7).

In the other types of GII phylogenetic tree, the 40 sporadic sequences were clustered into seven GII clusters $(2 / 5 / 6 / 8 / 13 / 14 / 21)$ and seven GII sub-clusters. In the other types of GII phylogenetic tree, about eight sporadic strains developed into nine outbreaks. In the GII.6 cluster, 15 sporadic sequences and four outbreak ones were subdivided into two sub-clusters (GII.6|Seacroft90/SaitamaU16). In the GII.2 cluster, four sporadic sequences and 95 outbreaks were sub-divided into three sub-clusters (GII.2|Akita7/CUHK-NS-1222/CUHK-NS-1224). 52 GII.2|CUHK-NS-1222 (KY677833) outbreak strains and 37 GII.2|CUHK-NS-1024 (KY677828) outbreak strains caused the GII.2 explosion in Seoul since November 2016. In the GII.21 cluster, 11 sporadic sequences were sub-divided into two sub-clusters (GII.21|Beijing-55185/ OC05024). The clusters GII.5, GII.8, and GII.14 were further divided into one sub-cluster (GII.5|Hillingdon90, GII.8|Amsterdam, and GII.14|Beijing) with only one sporadic sequence belonging to a sub-cluster. Although the GII.1 cluster and the GII.2|CUHK-NS-1024 sub-cluster included 17 and 37 outbreaks, respectively, they did not include any sporadic sequences (Figs. 3 and 8).

\section{Discussion}

To characterize phylogenetic epidemiology of $\mathrm{NoV}$ strains circulating in Seoul, we first conducted an extensive phylogenetic analysis based on partial VP1 sequences of total $1008 \mathrm{NoV}$ (794 NoV positives from $4073 \mathrm{AGE}$ specimens and 214 global references from NoroNet and GenBank). This extensive phylogenetic analysis revealed 


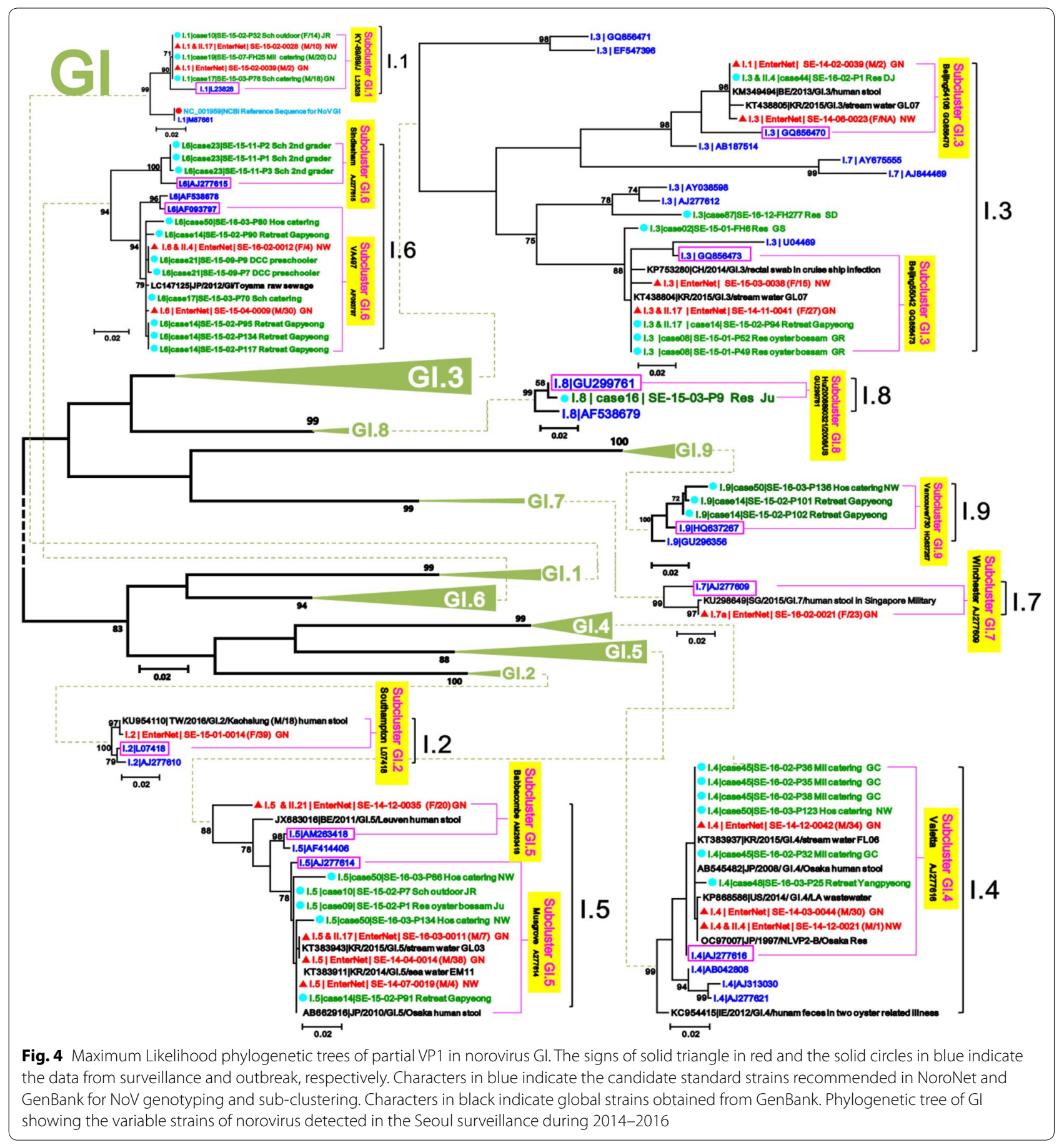

the monthly prevalence and distribution of NoV strains widespread in Seoul and their phylogenetic relationships with global reference strains. Through the five phylogenetic trees (GI/GII.4/GII.17/GII.3/other types of GII) obtained from the NoV-surveillance in 2014-2016, we could track emergences of the new strains and determine the trends of circulating strains in Seoul (Figs. 4, 5, 6, 7, $8)$.

In the GI phylogenetic tree, the three sporadic sequences (GI.4|Valetta, GI.3|Beijing55042 and GI.5|Musgrove) found in 2014 were identical to the nine outbreaks in 2015-2016. They were also identical to the global strains (AB545482 and KT383937 in GI.4|Valetta, 


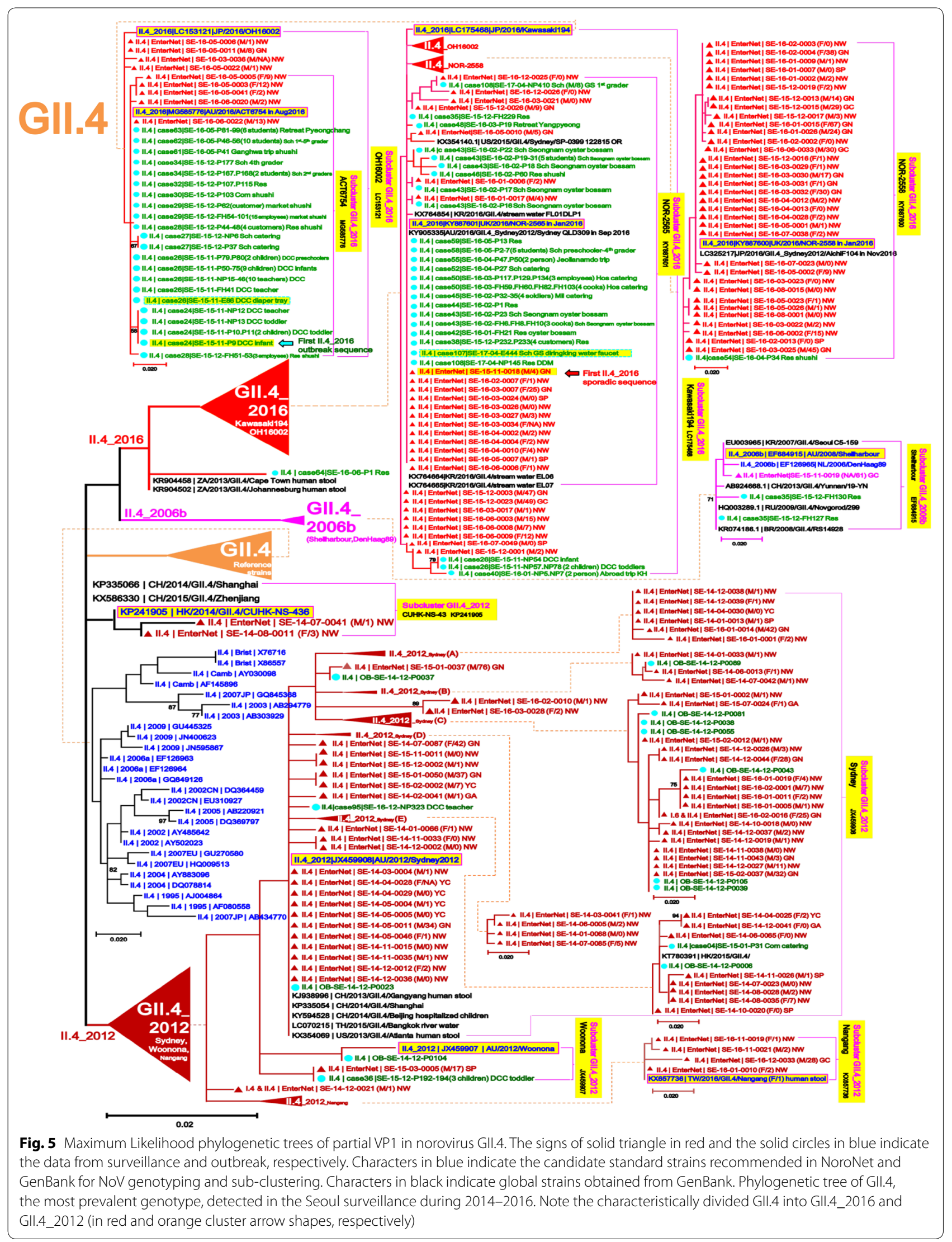




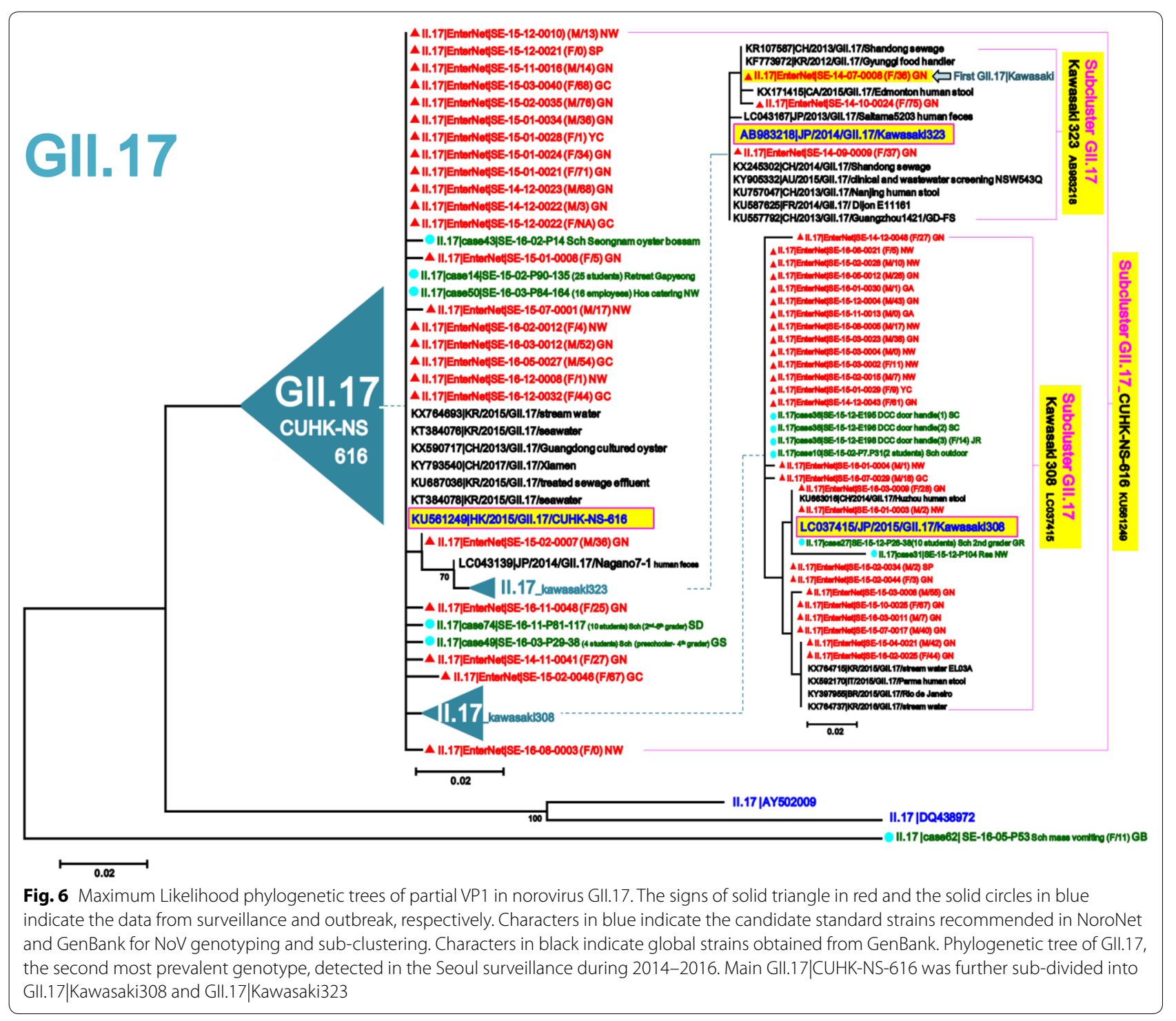

KT438804 in GI.3|Beijing55042, and AB662916 and KT383943 in GI.5|Musgrove) collected from human stools of Japan in 2008 and 2010, and from sea-water and stream-waters of Korea in 2014 and 2015 (Fig. 4). The data can suggest that the origin of Seoul sporadic GI strains may come from Japan.

In the three major phylogenetic trees of GII.4, GII.17 and GII.3, all the strains reported in the present study were the same as the global strains reported in NoroNet and GenBank. In the GII.17 phylogenetic tree, the first occurrence was detected as GII.17|Kawasaki323 (AB983218) in July 2014, which was identical to the new GII.P17-GII.17 reported in March 2014 in Japan [19]. The new GII.17 was $96 \%$ homologous in the amino acid sequences with the GII.17 strain reported in Korea by
KCDC [20]. Since first emergence of GII.17|Kawasaki323, GII.17|CUHK-NS-616 and GII.17|Kawasaki308 sharply increased during the winter in 2014-2015, and the GII.17 became the first dominant genotype in Seoul in January 2015 (Figs. 3 and 6). This epidemicity was well accordant with the global trend of the new GII.17 (GII.17|Kawasaki). According to the previous report by KCDC, the GII.17, previously considered as a minor type in Korea, is the predominant NoV since December 2014 [20]. During the winter in 2014-2015, the new GII.17 also emerged and became the predominant genotype in Japan [19], several major cities in mainland China [21, 22], and HongKong [23]. The new GII.P17-GII.17 was also detected sporadically outside of Asia such as Italy, Romania, and the United States [24-26]. In our previous 


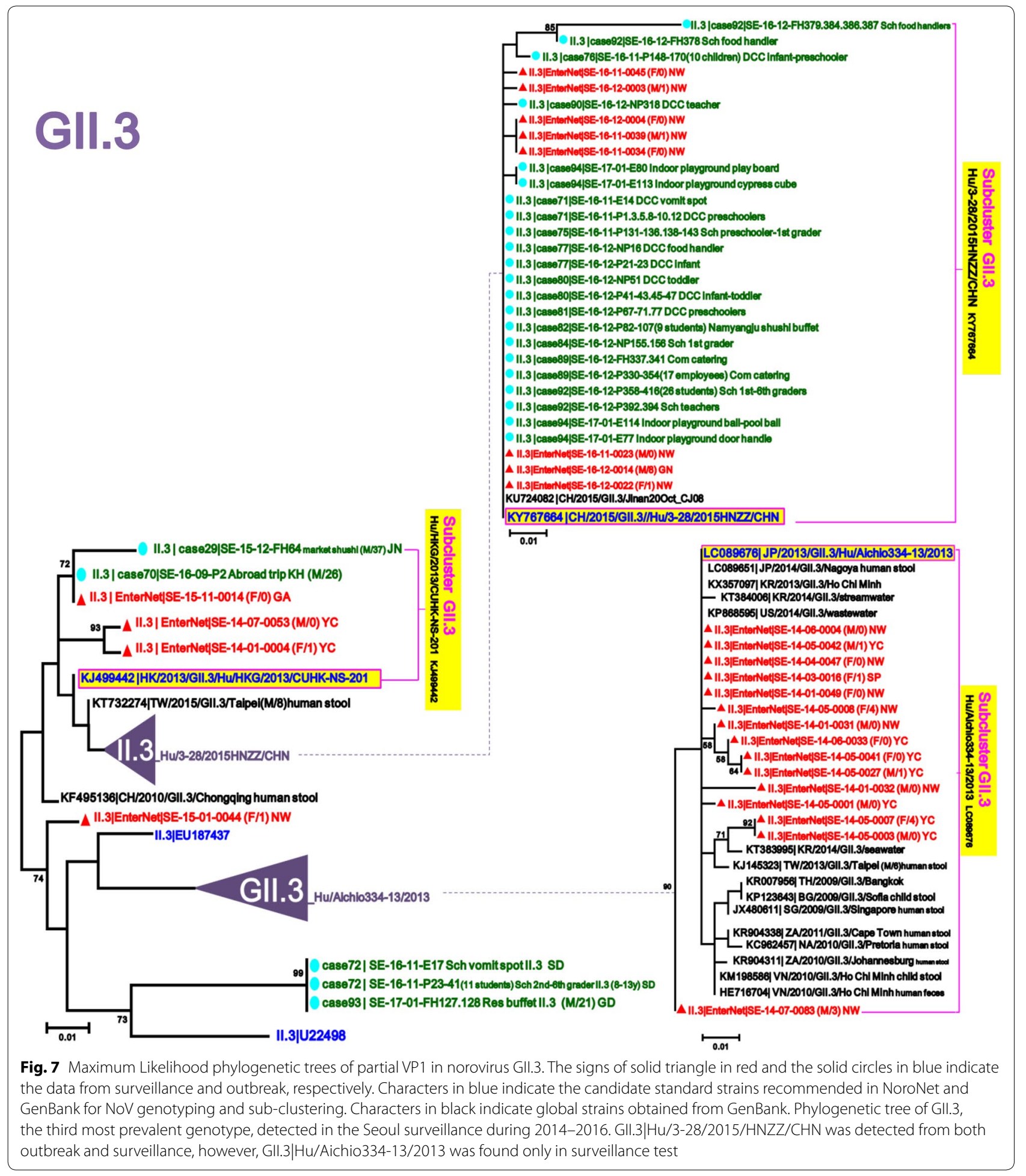

surveillance data during 2008-2011, 15 sporadic GII.17 were detected three times; 13 in March 2008, one in August 2008 and one in November 2010 [14]. The sporadic GII.17 in Seoul might be different from the past and current prevalent strains. The GII.17 detected in this study was presumed to be the new GII.17 that caused a pandemic since the winter of 2014.

In our sporadic data, GII.4 was still the most prevalent genotype (50.92\%, 138/271 positives) during the Seoul NoV-surveillance in 2014-2016 (Tables 2, 3 and Figs. 3, 
5). In the NoV GII.4 phylogenetic tree, all the sporadic GII.4 sequences were tightly clustered together, and largely sub-divided into two sub-clusters (GII.4|2012 and GII.4|2016) (Figs. 2 and 5). These sub-clusters showed distinct difference from the sporadic strains to outbreaks and seasonal epidemics. According to the previous report about GII.4|2012_Sydney, it was the most frequently found sub-genotype (60.4\%) during November 2012 and January 2013 in Korea [27]. GII.4|2012_Sydney was still the most frequently found sub-genotype (51.69\%, 46/89 positives) of the Seoul NoV-surveillance in 2014 (Fig. 5). Since November 2015, GII.4|2012 was replaced internally by novel GII.4|2016_Kawasaki194 (NOR-2565/NOR2558/OH16002) that was the variants of GII.4|2012 Sydney (JX459908). The sharply increased sporadic $\mathrm{NoV}$ in the winter of 2015 (Fig. 3) was mainly caused by II.4|2016_Kawasaki194 (LC175468) that was first detected from AGE patients in Kawasaki City in 2016 [28]. Since its first detection, GII.4|2016_Kawasaki194 spread very rapidly and caused sporadic NoV explosion, which raised the average NoV detecion rate from 13.17\% $(89 / 676$ samples in 2014$)$ to $20.61 \%$ (108/524 samples in 2016) during the present study period. Due to unusually high NoV activity, seasonal epidemic curve in 2016 was skewed to the novel "right-sided W-shaped" curve (Fig. 3). By reanalyzing the Seoul NoV-surveillance data in 2007-2013, we found that the epidemic curve in 2007 was similar with our novel "right-sided W-shape" curve in 2016 (dotted box in Fig. 3). Additional evolution studies are required to investigate why the estimated GII.4|2006b and GII.4|2016_Kawasaki194 strains spreaded more rapidly and caused heavy explosions in Seoul in 2007 and 2016 compared to other pandemic strains. In the II.4|2016_Kawasaki194 sub-cluster, our 11 sporadic sequences were identical to four global strains (KY887601, KY905335, KX764665 and KX764664), which were collected from human stools and stream waters in January and September 2016. Above-mentioned four global strains and the II.4|2016_Kawasaki194 strain were all reported as GII.P16-GII.4 Sydney2012 recombinants [29,30]. The first appearance of the Seoul sporadic Kawasaki194 strain was in November 2015, which is at least a few months ahead of identical global strains and the II.4|2016_Kawasaki194 candidate standard strain.
In our outbreak data, GII.3 and GII.2 exploded and caused huge outbreaks during the winter of 2016 (Figs. 7 and 8). Although sporadic GII.3 became the predominant genotype in last quarter of 2016 from the Seoul NoV-surveillance (Figs. 3 and 7), sporadic GII.2 remains low until December 2016 (Figs. 3 and 8). Although GII.3/Hu/3-28/2015/HNZZ/CHN strain caused heavy outbreaks in Seoul since November 2016, the most dominant strain in the Seoul NoVsurveillance was not GII.3/Hu/3-28/2015/HNZZ/ CHN but GII.3|Hu/Aichio334-13/2013 (Fig. 7). In the GII.3 phylogenetic analysis, we also observed that GII.3|Hu/Aichio334-13/2013, the dominant strain in 2014, could not develop outbreak in Seoul, whereas GII.3|Hu/3-28/2015/HNZZ/CHN rapidly developed outbreak. We, here, suggest that the sporadic GII.3|Hu/3-28/2015/HNZZ/CHN might be a GII. P16-GII.3 variant. Although sporadic GII.2 and GII.6 were detected at low prevalence during the whole study period, they are important genotypes prevalent in worldwide and should be carefully monitored [31,32].

\section{Limitation}

We agree with the presence of several limitations in the present study to determine the geographical distribution and mechanism of the NoV outbreak. However, this study was abided by the K-CaliciNet and the national norovirus surveillance guidelines [12]. The followings may explain potential reasons of our present limitation; First, present analysis of target sequences was confined to the partial VP1 region. Although it is not long enough to detect whole genome of NoV, the VP1 was designated as the NoV surveillance target sequences based on its high variability in sequences and efficiency. The sequencing of partial VP1 also allows large quantities of NoV to be genotyped economically with epidemiologic trends. A novel NoV lineage containing the GII.P16 polymerase and pandemic GII.4 Sydney and other GII capsid were recently detected in Asia and Europe during the winter in 2016 to 2017 [29]. To examine NoV evolutions in recombination and surface-exposed antigenic regions, future study should be focused on exploring the large target sequences covering RdRp and complete VP1 region. Second, the 10 hospitals employed for the present study were not evenly distributed in Seoul (three out of 10 hospitals

(See figure on next page.)

Fig. 8 Maximum Likelihood phylogenetic trees of partial VP1 in other types of norovirus Gll. The signs of solid triangle in red and the solid circles in blue indicate the data from surveillance and outbreak, respectively. Characters in blue indicate the candidate standard strains recommended in NoroNet and GenBank for NoV genotyping and sub-clustering. Characters in black indicate global strains obtained from GenBank. Phylogenetic tree of other types of Gll (2/5/6/8/13/14/21) showing variable strains of norovirus detected in the Seoul surveillance during $2014-2016$ 


\section{Other types of GII $(2,5,6,8,13,14,21)$}

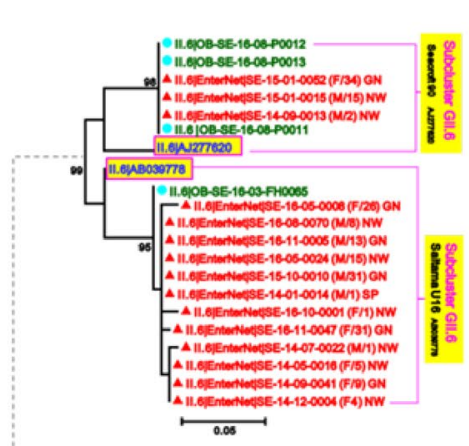

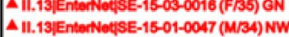
A II. 13 EntorNetsE-15-01-0039 (F/31) GN

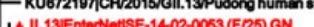
H 1.13 [E MTONOQSE-1402-0063 (F/25) GN A II.13] EnterNetSE-14-03-0010 (F/2B) GN -

- II.13|OB-SE-16-03P011

I 1 II.13|EnterNotse-15-02-0038 (M/35) GN
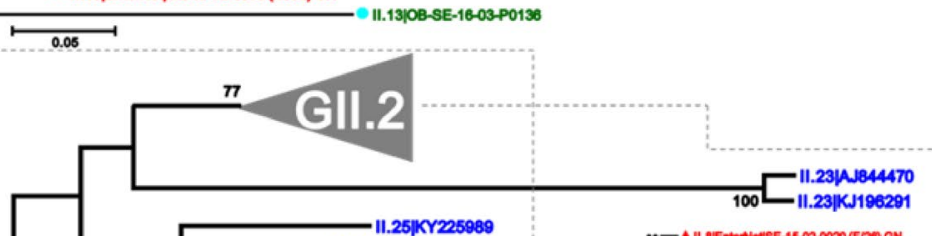

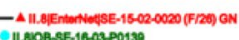

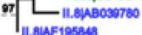
II. Q.MF

0.08

57- 11.14 AYY130781

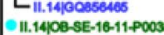

A II. 14 EntenNotisE-16-00-0073 (M/T) NW

-11.201AB542917

424333

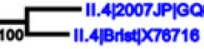

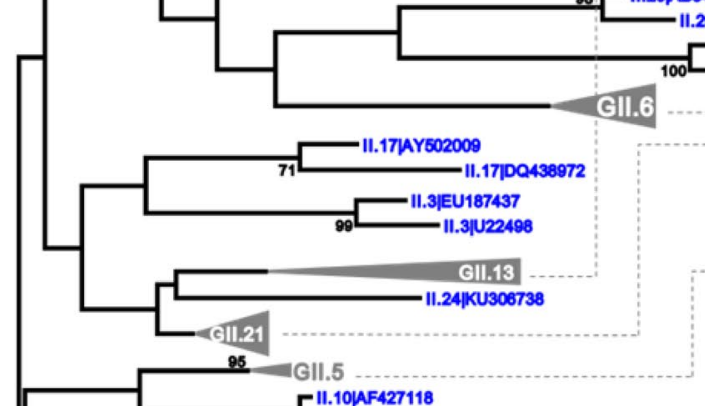

II.10/AF427118

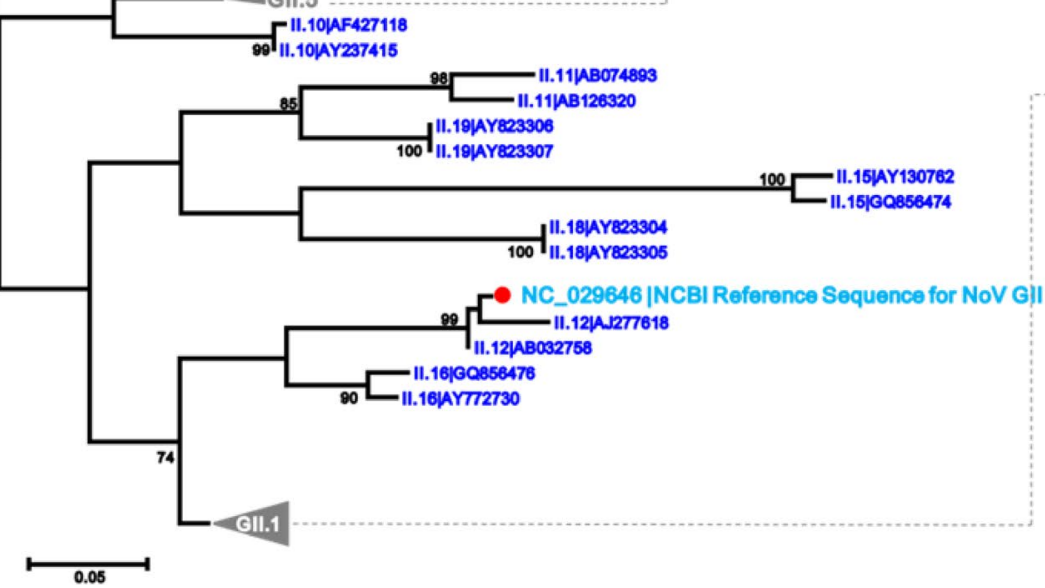

85 II.5IOB-SE-10-11-SE-16-02

II.5|OB-SE-16-12-P0024

III.6|EnterNet|SE-14-05-0012 (F/32) ON

0.05

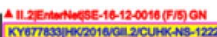

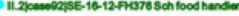

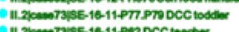

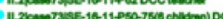

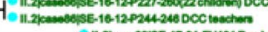

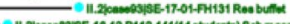

if

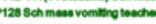

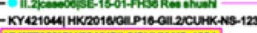

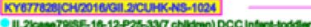

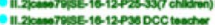

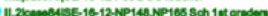

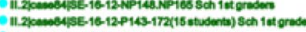

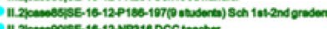

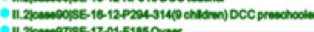

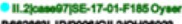

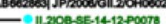

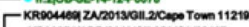

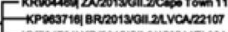

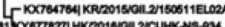

$1+12+0000$

11.2.200-SE 14.12P00000

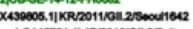

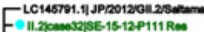

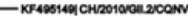

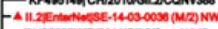

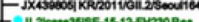

AII 2 IE

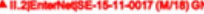

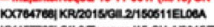

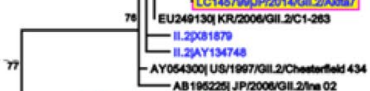

A 1900250

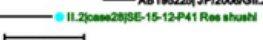

$\stackrel{0.08}{\longmapsto}$

[- II.21108-SE-16-03-FH0067

A II.21|EntarNotSE-14-12-0035 (F/20) ON

-A II.21|EntarNeySSE-15-08-0008 (M/3) NW

$\Delta$ II.21|EntorNotSE-16-05-0047 (F/O09) GN

$\Delta$ II. 21|EnterNotsSE-15-06-0002 (M/44) NW

$\Delta 11.21$ EnterNots

$\Delta$ 11.21|EnterNotsE-15-06-0030 (M/O) NW

- 11.21 1108-SE-15-07+0031

- $11.21108-2 E-15-08-P 0030$

L A 11.21|EntortiotsE-15-08-0007 (M/3) NW

- II.210085e460

11.21108-SE-16-03P0063

A II.21|EntorNetsE-16-02-0027 (M/28) GN

- II.21|OE-SE-16-03P0000

- 1.21)08-SE-16-03FHOONG

T.

11.21|OB-SE-16-03P0120

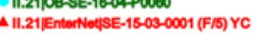

11.21)En

A.

$\longmapsto$

70 - II.11007611

OII1OBSE-1801P0000

II.1|OB-SE-16-01-P0009

II. 1|OB-SE-16-05-P0064
II.1|OB-SE-16-05-P0065

II. 1 |OB-SE-16-05-P0045

II. 1|OB-SE-16-05-P0043

II.1|OB-SE-16-05-P0042

91 CI.11OB-SE-15-12-P0202

II.11OB-SE-15-12-P0200

II.1|OB-SE-15-12P0204

II.1/OB-SE-15-12-P020

II.1|OB-SE-15-12-P0208

II.1/OB-SE-15-12-P0209

II.11OB-SE-15-12+0210

II.11OB-SE-15-12P0211

II.1|OB-SE-15-12-P0213

II.1|OB-SE-15-12+P0217

II.11OB-SE-15-12-P0200 
were localized in one administrative district-Gu) (Fig. 1). Although we could track new strains through phylogenetic analysis with outbreaks and global strains, it was insufficient to cover the detailed NoV transmission routes. Lastly, our sample collection was limited to the patients with symptomatic infection, however, over $30 \%$ of NoV infection is asymptomatic with shedding virus [33].

\section{Conclusions}

During 2014-2016, we determined 17 NoV genotypes and their sub-genotypes widespread in Seoul. By the first extensive phylogenetic characterization of 1008 specimens, we could track the emergence of new NoV strains that is able to cause massive outbreak or sporadic AGE infection globally. Most of them were found to be the novel variants of three major genotypes (GII.4, GII.17 and GII.3). Main epidemiologic event in the 2014-2016 Seoul NoV surveillance was continuous emergences of novel NoV strains of GII.17 (GII.17|2014_Kawasaki lineages in 2014), GII.4 (GII.4|2016_Kawasaki194 lineages in 2015), and GII.3 (GII.3|Hu/3-28/2015/HNZZ/ CHN in 2016). Our results demonstrate that emergent GII.4|2016_Kawasaki194 lineages spread throughout Seoul very rapidly and caused unusually heavy explosions, especially in Seoul in 2016 compared to other two pandemic strains (GII.4|Sydney and GII.17|Kawasaki).

By analyzing the development from sporadic strains to outbreaks in various phylogenetic trees, we can show distinctly different patterns depending on each NoV lineages. Our report has an important implication in the understanding $\mathrm{NoV}$ incidence and developing a treatment vaccine against NoV.

\section{Additional file}

Additional file 1: Table S1. Candidate standard strains for genotyping and sub-clustering.

\begin{abstract}
Abbreviations
AGE: acute gastroenteritis; NoV: norovirus; qRT-PCR: real-time reverse transcription polymerase chain reaction; RT-PCR: reverse transcription polymerase chain reaction; ORF: open reading frame; DB: Dobong; DDM: Dongdaemun; DJ: Dongjak; EP: Eunpyeong; GA: Gwanak; GB: Gangbuk; GC: Geumcheon; GD: Gangdong; GJ: Gangjin; GN: Gangnam; GR: Guro; GS: Gangseo; JN: Jungnang; Ju: Jung; JR: Jongno; MP: Mapo; NW: Nowon; SB: Seongbuk; SC: Seocho; SD: Seongdong; SDM: Seodaemun; SP: Songpa; YDP: Yeongdeungpo; YC: Yangcheon; YS: Yongsan; AU: Australia; BE: Belgium; BR: Brazil; CA: Canada; CH: China; HK: HongKong; Fin: Finland; IE: Ireland; IT: Italy; JP: Japan; KH: Cambodia; KR: Korea; NL: Netherlands; SG: Singapore; TH: Thailand; TW: Taiwan; UK: United Kingdom; US: United States; VN: Vietnam; ZA: South Africa; DCC: day-care center; Hos: hospital; Mil: military base; Res: restaurant; Sch: school.
\end{abstract}

\section{Authors' contributions}

YEK, MS, HS and EYK performed the experiments, YEK, JL, JY, YH, IK and TY examined the statistical analysis. YEK, IKL and JY wrote the manuscript. YEK conceived and designed the experiments, TJP and IKL edited the manuscript. All authors read and approved the final manuscript.

\section{Author details}

${ }^{1}$ Department of Infectious Disease Research, Seoul Metropolitan Government Research Institute of Public Health and Environment, 30 Janggunmaeul 3-gil, Gwacheon 13818, Republic of Korea. ${ }^{2}$ Department of Biochemistry and Molecular Biology, Ajou University School of Medicine and Graduate School of Medicine, 164 Worldcup-ro, Yeongtong-gu, Suwon 16499, Republic of Korea.

\section{Acknowledgements}

This research was supported by National Norovirus Surveillance System (K-CaliciNet) and Seoul Metropolitan Government Research Institute of Public Health and Environment.

\section{Competing interests}

The authors declare that they have no competing interests.

\section{Availability of data and materials}

The database supporting the conclusions of this article is included in the article's Additional file.

\section{Consent for publication}

Not applicable.

\section{Ethics approval and consent to participate}

Not required.

\section{Funding}

Not applicable.

\section{Publisher's Note}

Springer Nature remains neutral with regard to jurisdictional claims in published maps and institutional affiliations.

Received: 29 March 2018 Accepted: 19 August 2018

Published online: 27 August 2018

\section{References}

1. Liu L, Oza S, Hogan D, Chu Y, Perin J, Zhu J, Lawn JE, Cousens S, Mathers C, Black RE. Global, regional, and national causes of under-5 mortality in 2000-15: an updated systematic analysis with implications for the Sustainable Development Goals. Lancet. 2016;388(10063):3027-35.

2. Lopman B, Atmar R, Baric R, Estes M, Green K, Glass R, Hall A, IturrizaGomara M, Kang C, Lee B, Parashar U, Riddle M, Vinjé J. Global burden of norovirus and prospects for vaccine development. Atlanta: CDC. 2015;1-46.

3. Bok K, Abente EJ, Realpe-Quintero M, Mitra T, Sosnovtsev SV, Kapikian AZ, Green KY. Evolutionary dynamics of Gll.4 noroviruses over a 34-year period. JVirol. 2009;83(22):11890-901.

4. Glass RI, Parashar UD, Estes MK. Norovirus Gastroenteritis. N Engl J Med. 2009:361(18):1776-85.

5. Xi JN, Graham DY, Wang KN, Estes MK. Norwalk virus genome cloning and characterization. Science. 1990;250(4987):1580-3.

6. Cotten M, Petrova V, Phan MVT, Rabaa MA, Watson SJ, Ong H, Kellam P. Deep sequencing of norovirus genomes defines evolutionary patterns in an urban tropical setting. J Virol. 2014;88(19):1 1056-69 (PMID: 25056894).

7. Robert L, Atmar MD. Noroviruses-State of the Art. Food Env Virol. 2010;2(3):117-26.

8. Vinje J. Advances in laboratory methods for detection and typing of norovirus. J Clin Microbiol. 2015;53(2):373-381 (PMID: 24989606).

9. Bull RA, Eden JS, Rawlinson WD, White PA. Rapid evolution of pandemic noroviruses of the Gll.4 lineage. PLoS Pathog. 2010;6(3):1-10 (PMID: 20360972).

10. Siebenga JJ, Vennema H, Zheng D-P, Vinjé J, Lee BE, Pang X-L, Ho ECM, Lim W, Choudekar A, Broor S, Halperin T, Rasool NBG, Hewitt J, Greening 
GE, Jin M, Duan Z-J, Lucero Y, O'Ryan M, Hoehne M, Schreier E, Ratcliff RM, White PA, Iritani N, Reuter G, Koopmans M. Norovirus Illness is a global problem: emergence and spread of norovirus Gll.4 Variants, 2001-2007. J Infect Dis. 2009;200(5):802-12.

11. Boon D, Mahar JE, Abente EJ, Kirkwood CD, Purcell RH, Kapikian AZ, Green KY, Bok K. Comparative evolution of GII.3 and GIl.4 norovirus over a 31-year period. JVirol. 2011:85(17):8656-66 (PMID: 21715504).

12. Jung K. Guideline for water and foodborne diseases prevention and control. Korea: Korea Centers for Disease Control \& Prevention; 2016.

13. Park SH, Kim EJ, Yun TH, Lee JH, Kim CK, Seo YH, Oh SA, Choi SS, Cho SJ, Kim MS, Han GY, Kim MY, Jeong HS, Cheon DS, Kim HS. Human enteric viruses in groundwater. Food Environ Virol. 2010;2(2):69-73.

14. Ham H, Oh S, Kim C, Jang J, Jo S, Choi S, Information C. Molecular characteristics of human noroviruses genogroup I and genogroup || detected in acute gastroenteritis patients in Seoul. J Environ Health Sci. 2012;38(1):57-65

15. Ham H, Oh S, Seung H, Jo S. Molecular characteristics of noroviruses genogroup I and genogroup II detected in patients with acute gastroenteritis. Ann Lab Med. 2015;35:242-5 (PMID: 25729728).

16. Lysén M, Thorhagen M, Brytting M, Hjertqvist M, Andersson Y, Hedlund KO. Genetic diversity among food-borne and waterborne norovirus strains causing outbreaks in Sweden. J Clin Microbiol. 2009;47(8):2411-8 (PMID: 19494060)

17. Ando T, Noel JS, Fankhauser RL. Genetic Classification of "Norwalk-like Viruses". J Infect Dis. 2000;181 (Suppl 2):S336-48 (PMID: 19494060).

18. Kroneman $A$, Vega E, Vennema H, Vinjé J, White PA, Hansman G, Green K, Martella V, Katayama K, Koopmans M. Proposal for a unified norovirus nomenclature and genotyping. Arch Virol. 2013;158(10):2059-68 (PMID: 23615870)

19. Matsushima Y, Ishikawa M, Shimizu T, Komane A, Kasuo S, Shinohara M, Nagasawa K, Kimura H, Ryo A, Okabe N, Haga K, Doan YH, Katayama K, Shimizu H. Genetic analyses of Gll.17 norovirus strains in diarrheal disease outbreaks from december 2014 to March 2015 in Japan reveal a novel polymerase sequence and amino acid substitutions in the capsid region. Eurosurveillance. 2015;20(26):1-6 (PMID: 26159307).

20. Jung S, Hwang B, Jung H, Chung G, Yoo C, Lee D. Emergence of norovirus Gll.17-associated outbreak and sporadic cases in Korea from 2014 to 2015. Osong Public Health Res Perspect. 2017;8(1):86-90 (PMID: 28443229).

21. Lu J, Sun L, Fang L, Yang F, Mo Y, Lao J, Zheng H, Tan X, Lin H, Rutherford S, Guo L, Ke C, Hui L. Gastroenteritis outbreaks caused by norovirus Gll.17, Guangdong Province, China, 2014-2015. Emerg Infect Dis. 2015;21(7):1240-2 (PMID: 26080037).
22. Gao Z, Liu B, Huo D, Yan H, Jia L, Du Y, Qian H, Yang Y, Wang X, Li J, Wang $Q$. Increased norovirus activity was associated with a novel norovirus Gll.17 variant in Beijing, China during winter 2014-2015. BMC Infecti Dis. 2015;18(15):574 (PMID: 26678989).

23. Chan MC, Lee N, Hung TN, Kwok K, Cheung K, Tin EK, Lai RW, Nelson EA, Leung TF, Chan PK. Rapid emergence and predominance of a broadly recognizing and fast-evolving norovirus Gll.17 variant in late 2014. Nat Commun. 2015;6:10061.

24. Medici MC, Tummolo F, Calderaro A, Chironna M, Giammanco GM, De Grazia S, Arcangeletti MC, De Conto F, Chezzi C, Martella V. Identification of the novel Kawasaki 2014 Gll.17 human norovirus strain in Italy, 2015. Euro Surveill. 2015;20(35):30010 (PMID: 26530698).

25. Dinu S, Nagy M, Negru DG abriela, Popovici ED amian, Zota L, Oprişan G Molecular identification of emergent GII.P17-GIl.17 norovirus genotype, Romania, 2015. Euro Surveill. 2016;21(7):7-9 (PMID: 26924169).

26. Parra Gl, Green KY. Genome of emerging norovirus Gll.17, United States, 2014. Emerg Infect Dis. 2015;21 (8):1477-9 (PMID: 26196235).

27. Kim HS, Hyun J, Kim H, Kim J, Song W, Lee KM. Emergence of Gll.4 Sydney Norovirus in South Korea During the Winter of 2012-2013. J Microbiol Biotechnol. 2013;23(11):1641-3 (PMID: 24043126).

28. Matsushima Y, Shimizu T, Ishikawa M, Komane A, Okabe N, Ryo A, Kimura H. Complete genome sequence of a recombinant GII.P16-GII.4 norovirus detected in Kawasaki City, Japan, in 2016. Genome Announc. 2016;4(5):5-6 (PMID: 27795262).

29. Ruis C, Roy S, Brown JR, Allen DJ, Goldstein RA, Breuer J. The emerging GIl. P16-Gll.4 Sydney 2012 norovirus lineage is circulating worldwide, arose by late-2014 and contains polymerase changes that may increase virus transmission. PLoS One. 2017;12(6):e0179572 (PMID: 28662035).

30. Choi YS, Koo ES, Kim MS, Choi JD, Shin Y, Jeong YS. Re-emergence of a Gll.4 norovirus Sydney 2012 variant equipped with GII.P16 RdRp and Its predominance over novel variants of Gll.17 in South Korea in 2016. Food Env Virol. 2017;9(2):168-78 (PMID: 28120262).

31. Xue L, Wu Q, Kou X, Cai W, Zhang J, Guo W. Genome characterization of a Gll.6 norovirus strain identified in China. Infect Genet Evol. 2015;31:110-7 (PMID: 25660038)

32. Luo LF, Qiao K, Wang XG, Ding KY, Su HL, Li CZ, Yan HJ. Acute gastroenteritis outbreak caused by a Gll6 norovirus. World J Gastroenterol. 2015;21(17):5295-302 (PMID: 25954103).

33. Graham DY, Jiang X, Tanaka T, Opekun AR, Madore HP, Estes MK. Norwalk virus infection of volunteers: new insights based on improved assays. J Infect Dis. 1994;170(1):34-43 (PMID: 8014518).

\footnotetext{
Ready to submit your research? Choose BMC and benefit from:

- fast, convenient online submission

- thorough peer review by experienced researchers in your field

- rapid publication on acceptance

- support for research data, including large and complex data types

- gold Open Access which fosters wider collaboration and increased citations

- maximum visibility for your research: over $100 \mathrm{M}$ website views per year
}

At BMC, research is always in progress.

Learn more biomedcentral.com/submissions 